\title{
Comparative Analysis of Trauma Focused-Cognitive-Behavioral Therapy (TF-CBT) and Adlerian Therapy
}

\section{Aprille Woodson-Campbell*}

Argosy University Sarasota, USA

\begin{abstract}
Two counseling theories, Trauma-Focused Cognitive Behavioral Therapy and Adlerian Therapy are examined for their effectiveness as an integrative model in the treatment of patients who have experienced trauma. The integrative theoretical model is examined and conceptualized in the treatment of individuals, couples and families who have experienced traumatic situations.
\end{abstract}

Keywords: Integrative theoretical model; Trauma; Adlerian therapy; Trauma-focused cognitive behavioral therapy; Conceptualization; Symptoms

\section{Introduction}

Theory is the foundation of every therapeutic approach in the treatment of people with mental health disorders. Much focus has been placed on types of theories, methods, and treatments that best meet the needs of clients. There are two approaches that have served as effective therapeutic treatment for clients who suffer from a variety of conditions. Trauma Focused-Cognitive-Behavioral Therapy (TF-CBT) and Adlerian Therapy or Individual Psychotherapy has produced positive effects in the treatment of many disorders. TF-CBT is an approach subscribed to by this author based on recent practical experience and training over the past year in a clinical setting because of the positive results presented by clients who have suffered early onsets of trauma. The principles of cognitive behavior therapy and trauma treatment were originally designed to treat childhood trauma associated with sexual abuse. It is comprised of many integral parts that adapt to the developmental stages of children and individuals. For example, people who experience difficulty with certain aspects of their childhood are good candidates for this type of therapy. TFCBT protocol was developed for delivery over twelve sessions, but the therapist can adapt depending upon the need of the client. Although TF-CBT was designed to address childhood and adolescent traumas it is also considered effective in adults with a history of childhood traumas as well as current trauma. This treatment model is designed to treat traumas such as death, domestic violence, sexual abuse, divorce, loss of relationships, and generally any type of trauma an individual may experience. The protocol is designed so that children and their parents are treated simultaneously, separately or together. Clinicians must be trained to implement the model which incorporates components expressed by the acronym PRACTICE: "Psycho education and parenting skills, Relaxation skills, Affect expression and regulation skills, Cognitive coping skills and processing, Trauma narrative, In vivo exposure (when needed), Conjoint parent-child sessions and Enhancing safety and future development" [1]. Successful treatment is believed to have long-term effects even two-years after treatment [2].

TF-CBT is also effective in treating depression, posttraumatic stress disorder (PTSD), and other behavioral problems. In fact, TFCBT studies in clients with PTSD have proven effective in "reducing symptoms" as well as "depression, behavioral problems, shame" and other symptoms [3]. Empirical evidence supports the positive effects of treatment and coping skills when cognitive behavioral therapeutic interventions occur. According to Foa, Koane, Friedman and Cohen
[4] there are five principles that are critical to this approach, "(1) sense of safety; (2) promoting calming; (3) promoting a sense of self and community efficacy; (4) promoting connectedness; and (5) instilling hope" [5].

When analyzing, comparing, and integrating Adlerian Therapy in the TF-CBT model it seems to be a natural occurrence. Let's review; in Adlerian Therapy or Individual Psychology, Adler placed a high notion on environment and childhood development. How a child grows socially and environmentally will highly influence certain behaviors. Therefore, it is critical to examine any early trauma experiences a child or an adult who experienced trauma during childhood or throughout their lifetime. Adler believed the central concept to an individual's life is centered on what he called "style of life." He believed that a person's perception of themselves is influenced by their environment, and behavioral patterns are established by the age of 4 . Based on these influences and early-on patterns, one can ask if an individual has the ability to change; Adler believed so. According to Adler, there is a relationship between a person's style of life, creative power, and freedom of choice [6]. According to Butler and Newlon [7] who subscribe to Adler's notions on ones style of life and personality traits, certain children who experience trauma develop a specific perception of themselves and the world around them. For example, some children may believe people cannot be trusted. They may expect tragic things to occur in their lives and feel they have no control over the situation (Table 1).

The belief systems of children of trauma can strongly influence patterns of behavior, self-esteem, life choices, relationships with family, and relationships with others if these beliefs are not challenged in a structured, safe, and healthy way for the victim of a traumatic experience [8]. Because children have difficulty articulating their feelings and experiences, then these patterns of behavior form early in life resulting in maladaptive behaviors and beliefs about others as well as themselves. Research has revealed that children and even adolescents experience a

*Corresponding author: Aprille Woodson Campbell, Argosy University Sarasota, USA, Tel: 404-380-0422; E-mail: aprillecampbell@gmail.com

Received April 11, 2014; Accepted July 25, 2014; Published July 27, 2014

Citation: Campbell AW (2014) Comparative Analysis of Trauma Focused Cognitive-Behavioral Therapy (TF-CBT) and Adlerian Therapy. J Trauma Treat 3 : 201. doi: $10.4172 / 2167-1222.1000201$

Copyright: ( 2014 Campbell AW. This is an open-access article distributed under the terms of the Creative Commons Attribution License, which permits unrestricted use, distribution, and reproduction in any medium, provided the original author and source are credited. 


\section{Beliefs about Self}

"I expect bad things to happen to me."

"I want to be special and have others take an interest in me."

"I expect to be alone and abandoned."

"I expect to be misunderstood."

"I want others to understand me."

"I want to take care of others."

"I want to be excited."

\section{Beliefs about Others}

"People are untrustworthy and unwilling to be involved in my life."

"The world is a place filled with trouble and conflict."

"The world is unsafe."

"Life is dangerously frightening."

"Life is out of control."

Table 1: The chart, adapted from Butler and Newlon's [7] work, show some typically beliefs noted by these children [8].

"neuroanatomic" change in the brain when they have been through a traumatic experience. Turley and Obrzutm [9] explain that children suffering from trauma such as PTSD will often exhibit deficits during a neuropsychological assessment. They may have loss in memory, limits in their attention span, and intellectual verbal expression which should form disorders such as Attention Deficit Hyperactivity Disorder (ADHD) [9]. Therapist who use Adlerian techniques, particularly those techniques involved in play therapy have a higher success rate in treatment of children who have experienced trauma. Adlerian play therapy places great value on the client altering "faulty, self-defeating perceptions of self, others, and the world and move from these faulty convictions (private logic) to common sense" [10]. The goal of Adlerian play therapy is one of positive behavior while increasing the client's "sense of belonging". Comparatively speaking both TF-CBT and Adlerian Therapy seek to influence a positive change in behavior by examining behavioral patterns, promote a sense of safety and selfawareness, and alter faulty belief systems.

\section{Compatibility of Selected Theories for Integration}

Adlerian Therapy or better known as Individual Psychology and TF-CBT are compatible and appropriate for integration. Adler's theory examines and addresses an individual's life holistically, and strives for the individual to set goals and make positive changes. Adlerian therapist use techniques such as goal-setting, encouragement, confrontation, and "the Question" to empower clients to change behaviors and achieve a central purpose. TF-CBT focuses on the individual developing skills that will aid in changing the persons behavior. TF-CBT seeks to also empower clients to navigate through their traumatic experiences, while reducing their symptoms and giving them a sense of self-worth. While Adlerian Therapy and TF-CBT have uniquely different approaches, they are both effective in the treatment of mental health disorders, particularly traumas that have crippled many people in making the necessary changes needed to live an emotionally healthy life.

\section{Integrative Theoretical Model for Conceptualization and Treatment of Individuals, Couples, and Families}

\section{TF-CBT theory applies to individuals}

This theory applies to individuals who have significant emotional and psychological symptoms related to trauma. Studies support positive outcomes in treatment that are more effective than other therapeutic approaches. TF-CBT is very effective for individuals who have experienced multiple traumas. It is especially effective in reducing and managing posttraumatic stress disorder, reactions triggered by memories, reminders of the trauma where "maladaptive coping such as avoidance" surfaces, issues of trust, shame and social competence [11].

\section{TF-CBT applies to couples}

TF-CBT applies to couples when it comes to infidelity, separation or divorce. However, for a closer look at this model, let's examine the area of infidelity as an interpersonal trauma. Initial findings by Baucom, et al. [12], "Both clinical observations and empirical investigations demonstrate that the discovery of an affair can have an overwhelming and devastating impact on a couple. Injured partners often report intense emotions that vacillate between rage toward the participating partner and inward feelings of shame, depression, overwhelming powerlessness, victimization, and abandonment [13-18]. Taken as a whole, many of these emotional, cognitive, and behavioral responses parallel the criteria for post-traumatic stress disorder. Therefore, conceptualizing the response to an affair as a reaction to an interpersonally traumatic event aids in the formulation of these difficult cases and the conduct of treatment [19-21]. Treatment in cases like this would direct the focus on the trauma, but raise the issue of forgiveness. The injured party needs to gain understanding and some sort of control whereby their world becomes clearer. The injured party and the offending party should both work through a process of forgiveness so each can come to an understanding of how they got to the place of infidelity. Baucom et al. [22] further explain that forgiveness as a treatment is consistent with: "(1) gaining a more balanced view of the offender and the event; (2) decreasing negative affect toward the offender, potentially along with increased compassion; and (3) giving up the right to punish the offender further."

\section{TF-CBT applies to families}

TF-CBT applies to families and is effective in empowering families to work with child/victims of trauma or abuse. TF-CBT also is effective in reducing symptoms families, parents, and caregivers may feel, including posttraumatic stress disorder and depression. In addition to many regions in the United States and the September $11^{\text {th }}$ tragedies, TF-CBT has been used to successfully treat families in countries such as Pakistan, Zambia, and Russia. The assessment process is critical to proper interventions for this approach. Assessments such as PTSD Diagnostic Scale (PDS); Beck Depression Inventory II (BDI-II); UCLA PTSD Index-Parent Version, Child Behavior Checklist (CBCL) are used to assess and treat parents, caregivers, and families. A randomized trial of TF-CBT compared to Child Centered Therapy (CCT) ran "among children whose uniformed service parents died in the September 11th terrorist attacks in New York City. Due to the service requirements of the funding agency, this project did not require clinical levels of CTG in participants. At pretreatment children did not have clinically significant levels of CTG or other outcome measures and no differences were found between the two treatment groups in outcomes at posttreatment. Mothers participating in this project did have clinically significant levels of PTSD, depression and general psychopathology at pretreatment, and those mothers who participated with their children in receiving TF-CBT experienced significantly greater improvement in all of these domains than those receiving CCT. Randomized trials of children with clinically significant levels of CTG are needed to further evaluate the efficacy of TF-CBT" [22].

Families are taught skills to address anxiety management, 
psycho education, trauma narrations, parenting skills and behavioral management, and coping skills. For example, one such intervention includes the Child and Family Traumatic Stress Intervention (CFTSI). CFTSI targets two risk factors, "poor social or familial support, and poor coping skills in the aftermath of potentially traumatic events" [23]. The primary goal includes an increase in communication between the child and family concerning feelings, symptoms, and behaviors. The focus here is on the caregivers increasing support of the child. Additionally, this intervention teaches special behavioral skills to families and children to aid them in coping with "traumatic stress reactions".

\section{Adlerian Therapy Applies to Individuals and Families}

Who have experienced trauma during childhood and throughout adulthood. Adlerian therapy has fortified the relationship between cognitive and social theories as a practical approach to treating trauma. This type of therapy adds a healthy balance by analyzing individual and correlating relationship such as family and friends. Adlerian therapy presents a "pragmatic approach that is flexible and uses a range of action-oriented techniques to explore personal problems within their sociocultural context" [24,25]. Additionally, this form of therapy seeks to closely examine internal and external factors and symptoms that persist in a social context. Adlerian therapist are able to examine an individual's "style of life" as a basis of understanding the pattern of behavior that exists at the time of the traumatic experience and prior to that experience. This is important in helping clients process the traumatic experience, while establishing appropriate patterns of behavior.

\section{Integrative Model with Ethical Application and Multicultural/Diversity Components}

The ethical application of treatment is a consideration all therapists must weigh when working with various groups and cultures. The appropriate standards and practices should not only be explored, but incorporated within a treatment regimen for mental health disorders. Therapist should be competent in the treatment of TFCBT and Adlerian therapy when exploring these two modalities. This includes the proper education, training, supervision, and peer-review consultations that will properly prepare the therapist in administering this approach.

When using a TF-CBT and Adlerian approach, ethical guidelines and certain multicultural competencies should be considered in order to meet the needs of the client/patient. Some of these competencies include cultural heritage, ethnic and racial identity, knowledge of assessment tools and procedures used for specific cultures, and the therapists "ability to accurately self-assess one's multicultural competence, including knowing when circumstances (e.g., personal biases; stage of ethnic identity; lack of requisite knowledge, skills, or language fluency; sociopolitical influences) are negatively influencing professional activities and adapting accordingly (e.g., obtaining needed information, consultation, or supervision or referring the client to a more qualified provider [26-29]. Therefore, it's important when assessing a client that diagnostic instruments be culturally centered to racial dynamics and frequent expressions used in their community. According to Sue, Gallardo, and Neville [29] "specific treatment strategies should be a culturally syntonic fit with the client's sociocultural experience, identities, and sensibilities. Treatment should be informed by examining how the intersections of person, culture, and context, and the congruence (or incongruence) between them, contribute to... their internally experienced and externally expressed distress." It's also important to note that cases where the interrelationship with "cultural" identity and "personal and psychological characteristics, and relevant contextual considerations" should be important considerations during treatment.

It's is key for therapist to understand cultural competencies when working with clients and examine their strengths and weaknesses. This will serve an important role in determining the continuation of a counselor services or referring the client out to a more culturally competent counselor. When examining culture from a TF-CBT and Adlerian approach, counselors should understand the contributory factors that led to the trauma. These factors must be considered in the context of a person's background and culture. A multicultural approach to therapy will effectively augment any other integrative therapeutic approach for many clients.

When conceptualizing clients who suffer from trauma such as a childhood sexual abuse, a full diagnostic and historical assessment of how childhood sexual abuse is viewed within the culture or community. The therapist should investigate any cultural barriers that may contribute to the client's thoughts about their culture and any inward expression or beliefs about the abuse. The therapist should analyze the client's belief system and thoughts.

Intervention for Trauma with Symptoms of Depression should include:

1. A Cultural Genogram to identify the client's family structure and relationships. This will help the client conceptualize the issues and patterns noted in the family.

2. Continual Feedback from the client will fortify their relationship with the therapist and help the client recognize if the therapist in understanding cultural needs. For example, "I think my counselor understands me and respects my cultural beliefs."

3. Establish goals that will address behavioral issues and cultural needs. These goals can be short-term or long-term.

4. Develop an awareness of how childhood issues have affected and continue to affect one's family life, i.e., childhood sexual abuse [30].

5. Resolve past childhood/family issues, leading to less anger and depression, greater self-esteem, security, and confidence [30].

The ultimate goal is improving the client's current conditions and providing him or her the cultural understanding and therapeutic approach needed to address their issues.

\section{Examination of a Fictional Case Vignette: Applying TF- $\mathrm{CBT}$ as an Integrative Model}

\section{Conceptualization of rachel using TF-CBT}

Case Conceptualization: For purposes of this case conceptualization, only one therapeutic model (TF-CBT) will be examined. Rachel is a 60 year old Caucasian-American female who sought in-patient treatment for severe depression, anxiety, and rug dependency. She first experienced cocaine at the age of 22 years old; however, she has not taken drugs for the past ten-years. She also has a history of sexual abuse as a child by her uncle. Her family history includes drug-addicted parents who were never married and drugaddicted siblings (four brothers and three sister). She comes from a poor economic background where she was bounced between her 
mothers' home in a small town in rural Kansas to her Aunt Julia's home as a young toddler until age 6 .

Presenting Concern and Brief History: Rachel voluntarily came into care due to her severely depressed mood. During her initial assessment she appeared severely depressed. She explained that she was depressed due to the death of her husband and brother. Rachel was married to her husband for twenty (20) years. She has a history of emotional abuse and feelings of abandonment by her parents, and her husband who he is deceased. Rachel has three children from a previous marriage. Her former spouse was physically and emotionally abuse towards her. Rachel explained she has been depressed most of her life (since her early childhood trauma of molestation).

Rachel expressed feeling deep pain due to the separation and eventual loss of her husband as a result of pancreatic cancer in January 2013. She says her feeling of pain is still fresh due to the death of her husband, and she recently lost her Aunt Maria, her oldest brother in a car accident, and a close friend as a result of a stroke. Rachel reports a family history of alcohol and substance abuse. She says her aunts, uncles, parents and siblings have struggled with either alcohol or drug abuse and she wishes to break the family cycle. Furthermore, she explains the triggers that led to her pattern of behavior resulting in low self-esteem and drug use. Rachel shared she was molested by her uncle as a child, and her mother was aware of the violation. Rachel explained that her mother never protected her, and she always put her down. She called her names such as "trash" and told her she would "never amount to anything." Rachel shared that she feels isolated and alone. She feels like she is the cause of her problems, and she further explains, without the support of her husband, she has no one to turn to because her children don't understand her.

Rachel reports a past history of depression, child molestation, verbal abuse, and drug dependency. She denies being bullied as a child or having thoughts of suicide. She said she "used drugs because of my depression." She also classified herself as "depressed" with low self esteem; this is due largely to her mother calling her names throughout her life. Her husband also called her names during their twenty years of marriage. Although her husband called her many names, she expressed having feelings of love for him, and appears to be grieving heavily for her loss.

Rachel has no history of suicidality or homocidality at the time of her initial assessment or at any point thereafter. She has seen a psychologist before for depression, but has no long history of treatment. Although she reports a past history of drug use versus abuse, she did not seek treatment for her use because she says she simply stopped.

When conceptualizing Rachel along the lines of trauma-focused cognitive-behavioral therapy it's important to briefly look at her history and background.

\section{Any Factors Affecting Counseling}

Patient has been in treatment for approximately a month; however, she exhibits deep depression and very low self-esteem. She explained she has been depressed all of her life, even in early childhood, and it has been difficult managing her depression. She reports during her twenty-year marriage, her husband "often" verbally abused her and called her names. Patient appears to have difficulty understanding why her husband behaved the way he did, and often blamed herself for his actions. Patient's own self-image and self-worth has posed as a barrier to her treatment and recovery. Patient reported increased depression and anxiety often, and has not been able to successfully overcome her depression. Patient reports her current boyfriend as verbally abusive, and says he can get confrontational at time. The patient continues to have a difficult time with her depressed mood and anxiety.

\section{DSM Multi-Axial Diagnostic Impressions with Justification}

\section{I. $\quad 995.53$ Sexual Abuse of Child, Victim}

296.3x Major Depressive Disorder, Recurrent

300.02 Generalized Anxiety Disorder

304.20 Cocaine Dependence

II. V71.09 No diagnosis

III. None

IV. Poor relationship with mother and sister; Feelings of poor self-esteem and worthlessness.

\section{V. $\mathrm{GAF}=10$ (at assessment)}

\section{$\mathrm{GAF}=50$ (current)}

Under Axis I, patient received multiple diagnostic specifiers from the DSM-IV-TR (2000) due to patients documented history of depression, trauma, and abuse.

No personality disorders appear to be present, so there is no justification for any Axis II diagnoses. There are no medical conditions reported by patient on Axis III.

Patient reports a severely impaired relationship with her biological mother that includes a host of emotional psychosocial problems under Axis IV.

Finally, the Global Assessment of Functioning (GAF) score of 10 was given at assessment because Rachel's symptoms were classified as below moderate. Her GAF increased slightly as she continued treatment because her desire to make progress. The inpatient treatment facility assigned her a GAF of 20 upon her admission there. She is currently continuing treatment, but expected to discharge from that facility; currently a GAF of 50 is appropriate because some symptoms, deep depression have improved at time. These GAF scores seem to be appropriate according to the DSM-IV-TR's descriptions of the GAF ranges.

\section{Case Conceptualization and Intervention based on Trauma Focused Cognitive-Behavioral Therapy Approach}

Many different therapeutic techniques are utilized in treatment of those with severe depressed mood and childhood trauma. Typically an approach of cognitive-behavioral therapy has been used to address these conditions. However, TF-CBT is recommended for treatment in cases such as this. As identified earlier in this paper, TF-CBT is based on principles of cognitive behavior therapy and trauma treatment. It was originally designed to treat childhood trauma associated with sexual abuse. Because Rachel has experienced sexual trauma in her earlier life TF-CBT would be an effect way to address her issues. It consists of many component parts that are tailored for children and family members. Although Rachel is not a child, she is having difficulty with certain aspects of her childhood when addressing her trauma. Effective treatment for Rachel could occur in as little as twelve sessions; however, she may require more sessions depending on her needs. TFCBT is also effective in treating Rachel's depression, trauma, chemical dependence, and other behavioral concerns that have inhibited her 
from advancing in personal and family relationships. Art-therapy, as an integrative approach through Adlerian techniques can also be integrated into Rachel's treatment. This is an effective form of treatment for childhood trauma.

When conceptualizing this particular case along the lines of trauma-focused cognitive-behavioral therapy the clinician should examine relevant cognitions that the patient had as she entered into therapy and as therapy progressed. The clinician should determine if the patient is experiencing vague memories of inappropriate childhood sexual contact that can be corroborated by others. Is the patient's selfreport of being sexually abused clear, with detailed memories? Does the patient have an inability to recall years of childhood? Does the patient have extreme difficulty becoming intimate with others? Whether there is an inability to enjoy sexual contact with a desired partner. Does the patient experience unexplainable feelings of anger, rage, or fear when coming into contact with a close family relative? Is there a pervasive pattern of promiscuity or the sexualization of relationships?

These questions are important in resolving issues of being sexually abused with an increased capacity for intimacy in relationships. The patient needs to understand. It's okay to begin the healing process from sexual abuse with resultant enjoyment of appropriate sexual contact. Ultimately, the patient can work through the issues related to being sexually abused with a feeling of control and power. She can recognize and accept the sexual abuse without inappropriate sexualization.

Initial short-term treatment includes the clinician building a trusting relationship with the patient while carefully exploring the sexual abuse. Perhaps have the patient engage in drawing a diagram of her family structure, including where family members slept. Exploring supportive relationships, or individuals who would provide encouragement to the patient, and supplement the sessions with the clinician with referrals to support groups for survivors of sexual abuse.

\section{Intervention for severe depression}

The long-term plan of treatment for patients like Rachel with severe depression involves an assessment of depressive thoughts and tendencies. The goal for Rachel is to alleviate depressed mood and return to a level of effective functioning. Therapy should work towards Rachel recognizing, accepting, and coping with feelings of depression. She should develop healthy cognitive patterns and beliefs about herself and the world that lead to alleviation and help prevent the relapse of depression symptoms. She should also develop healthy interpersonal relationships that lead to alleviation of symptoms and help prevent the relapse of depression. Finally, she should appropriately grieve the loss of her husband and aunt in order to normalize mood and to return to an adaptive level of functioning.

\section{Intervention for chemical dependence}

Behaviors of an individual with chemical dependence include a consistent use alcohol or other mood- altering drugs until the individual is high, intoxicated, or passed out. This individual is unable to stop or cut down use of mood- altering drug once started, despite the verbalized desire to do so and the negative consequences continued use brings. Rachel denies a history of drinking alcohol excessively, but she has a history of taking drugs to mask her depression. Rachel should complete an objective test of drug abuse. The clinician should process the results with Rachel. She should also be referred for a thorough physical examination to determine any physical or medical consequences of her chemical dependence. The clinician should arrange for an evaluation for a prescription of psychotropic medications such as serotonergic medications. She should be assigned to ask two or three people who are close to her to write a letter to the therapist in which they each identify how they saw the patient's chemical dependence negatively impacting her life. Rachel should complete a First Step paper and then process it with group, a sponsor or her therapist to receive feedback.

\section{Conclusion}

TF-CBT and Adlerian therapy are effective treatment plans for PTSD, clinical settings, as well as a teaching model for current and future counselors. It provides useful tools, assessment and treatment strategies, and role play for the treatment of children, adolescents, and adults who experience trauma. TF-CBT and Adlerian therapy give counselors insight into understanding and treating the many layers of trauma while providing a safe and ethical approach in helping patients manage their emotions due to traumatic occurrences.

\section{References}

1. SAMSHA (2014) Trauma-Focused Cognitive Behavioral Therapy. SAMSHA's National Registry of Evidence-based Programs and Practices, U.S. Department of Health and Human Services.

2. Cohen JA, Deblinger E, Mannarino AP, Steer RA (2004) A multisite, randomized controlled trial for children with sexual abuse-related PTSD symptoms. See comment in PubMed Commons below J Am Acad Child Adolesc Psychiatry 43: $393-402$.

3. Cohen JA, Berliner L, March JS (2000) Treatment of children and adolescents In Foa EB, Keane TM, Friedman MJ (Eds.) Effective treatment for PTSD 106138

4. Foa EB, Koane TM, Friedman MJ, Cohen JA (2009) Effective Treatments for PTSD: Practice Guidelines from the International Society for Traumatic Stress Studies, 2nd Ed.The Guilford Press.

5. Hobfoll S, Watson P, Bell CC, Bryant RA, Brymer MJ, et al. (2009) Five Essential Elements of Immediate and Mid-Term Mass Trauma Intervention: Empirical Evidence. Spring 7: 2.

6. Slavick S, Carlson J (2006) The Theory of Individual Psychology.Taylor \& Francis Group.

7. Butler TL, Newlon BJ (1992) Children of trauma: Adlerian personality characteristics. Individual Psychology: The Journal of Adlerian Theory, Research \& Practice 48: 313

8. Burgi Mueller VR (2012) Integrating Adler's Individual Psychology into Current Trauma Models: A Research Paper, Adler Graduate School.

9. Turley MR, Obrzut JE (2012) Neuropsychological Effects of Posttraumatic Stress Disorder in Children and Adolescents. Canadian Journal of School Psychology 27: 166-182.

10. Malchiodi CA (2005) Expressive Therapies. The Guilford Press 148

11. The National Child Traumatic Stress Network (2008) How to Implement Trauma-FocusedCognitive Behavioral Therapy (TF-CBT) 9

12. Baucom DH, Gordon KC, Snyder DK, Atkins DC, Christensen A (2006) Treating Affair Couples: Clinical Considerations and Initial Findings. Journal of Cognitive Psychotherapy: An International Quarterly 20: 375-392.

13. Abrahms Spring J (with Spring, M.) (1996) After the affair: Healing the pain and rebuilding trust when a partner has been unfaithful. New York: Harper Collins.

14. Brown E (1991) Patterns of infidelity and their treatment. New York: Brunner/ Mazel. Cano,A., \& O'Leary, K. D. (2000). Infidelity and separations precipitate major depressive episodes and symptoms of nonspecific depression and anxiety. Journal of Consulting and Clinical Psychology, 68, 774-781.

15. Gordon KC1, Baucom DH, Snyder DK (2004) An integrative intervention for promoting recovery from extramarital affairs. See comment in PubMed Commons below J Marital Fam Ther 30: 213-231.

16. Lusterman DD (1998) Infidelity: A survival guide. Oakland, CA: New Harbinger

17. Pittman E (1989) Private lies: Infidelity and the betrayal of intimacy. New York: Norton. 
Citation: Campbell AW (2014) Comparative Analysis of Trauma Focused - Cognitive-Behavioral Therapy (TF-CBT) and Adlerian Therapy. J Trauma Treat 3: 201. doi:10.4172/2167-1222.1000201

Page 6 of 6

18. Reibstein J, Richards M (1993) Sexual arrangements: Marriage and the temptation ofinfidelity. New York: Scribner's

19. Baucom DH, Gordon KC, Snyder DK (2005) Treating affair couples: An integrative approach. In J. L. Lebow (Ed.), Handbook of clinical family therapy. New York: Wiley 431-463.

20. Glass S, Wright T (1997) Reconstructing marriages after the trauma of infidelity. In W. K.Halford \& H. J. Markman (Eds.), Clinical handbook of marriage and couples interventions. Chichester, UK: Wiley 471-507.

21. Gordon KC, Baucom DH (1998) Understanding betrayals in marriage: a synthesized model of forgiveness. See comment in PubMed Commons below Fam Process 37: 425-449.

22. Brown EJ, Goodman RF, Cohen JA, Mannarino AP (2004) Randomized controlled treatment outcome study for childhood traumatic grief. Paper presented at Conceptualization, Measurement and Treatment of Childhood traumatic Grief Symposium, 20th Annual Meeting of the International Society for Traumatic Stress Studies, New Orleans, LA.

23. The National Child Traumatic Stress Network, (2012). Trauma-Informed Interventions, April issue 1.

24. Arciniega GM, Newlon BJ (1999) Counseling and psychotherapy: Multicultura considerations. In Capuzzi D, Gross DF (Eds.), Counseling \& psychotherapy: Theories and interventions. Upper Saddle River, NJ: Merrill/Prentice Hall 435 458.

25. Watts RE (2003) Adlerian Therapy as a Relational Constructivist Approach. The Family Journal: Counseling and Therapy for Couples and Families 11: 144

26. American Psychological Association (1993) Guidelines for providers of psychological services to ethnic, linguistic, and culturally diverse populations. American Psychologist 48: 45-48.

27. Enns C (1997) Feminist theories and feminist psychotherapies: Origins, themes, and variations. New York: Haworth Press. Enns, C. (1997). Feminist theories and feminist psychotherapies: Origins, themes, and variations. New York: Haworth Press.

28. Olkin R (1999) What psychotherapists should know about disability. New York: Guilford Press.

29. Sue DW, Arrcdondo P, McDavis RJ (1992) Multicultural counseling competencies and standards: A call to the profession. Journal of Counseling and Development 70: 477-486.

30. Jongsma AE, Peterson LM, Bruce TJ (2013) The Complete Adult Psychotherapy Treatment Planner, Fourth Edition. John Wiley \& Sons, inc 62-63. 